\title{
A multi-wavelength study of a double intermediate-mass protostar - from large-scale structure to collimated jets
}

\author{
J. Forbrich ${ }^{1,2,3}$, Th. Stanke ${ }^{4,5}$, R. Klein ${ }^{6,7}$, Th. Henning ${ }^{8}$, K. M. Menten ${ }^{1}$, K. Schreyer ${ }^{2}$, and B. Posselt ${ }^{2,3,6}$ \\ 1 Max-Planck-Institut für Radioastronomie, Auf dem Hügel 69, 53121 Bonn, Germany \\ 2 Astrophysikalisches Institut und Universitäts-Sternwarte Jena, Schillergäßchen 2-3, 07745 Jena, Germany \\ ${ }^{3}$ Harvard-Smithsonian Center for Astrophysics, 60 Garden Street, MS 72, Cambridge, MA 02138, USA \\ e-mail: jforbrich@cfa.harvard.edu \\ 4 Institute for Astronomy, University of Hawaii, 2680 Woodlawn Drive, Honolulu, HI 96822, USA \\ 5 European Southern Observatory, Karl-Schwarzschild-Str. 2, 85748 Garching bei München, Germany \\ 6 Max-Planck-Institut für extraterrestrische Physik, Giessenbachstr. 1, 85748 Garching bei München, Germany \\ 7 Department of Physics, University of California, Berkeley, CA 94720, USA \\ 8 Max-Planck-Institut für Astronomie, Königstuhl 17, 69117 Heidelberg, Germany
}

Received 14 July 2008 / Accepted 25 September 2008

\begin{abstract}
Aims. The earliest stages of intermediate- and high-mass star formation remain poorly understood. To gain deeper insights, we study a previously discovered protostellar source that is deeply embedded and drives an energetic molecular outflow.

Methods. The source, UYSO 1, located close to IRAS 07029-1215 at a distance of about $1 \mathrm{kpc}$, was observed in the (sub)millimeter and centimeter wavelength ranges, as well as at near-, mid-, and far-infrared wavelengths.

Results. The multi-wavelength observations resulted in the detection of a double intermediate-mass protostar at the location of UYSO 1. In addition to the associated molecular outflow, with a projected size of $0.25 \mathrm{pc}$, two intersecting near-infrared jets with projected sizes of $0.4 \mathrm{pc}$ and $0.2 \mathrm{pc}$ were found. However, no infrared counterparts to the driving sources could be detected in sensitive near- to far-infrared observations (including Spitzer). In interferometric millimeter observations, UYSO 1 was resolved into two continuum sources with high column densities $\left(>10^{24} \mathrm{~cm}^{-2}\right)$ and gas masses of $3.5 M_{\odot}$ and $1.2 M_{\odot}$, with a linear separation of $4200 \mathrm{AU}$. We report the discovery of a $\mathrm{H}_{2} \mathrm{O}$ maser towards one of the two sources. Within an appropriate multi-wavelength coverage, the total luminosity is roughly estimated to be $\lesssim 50 L_{\odot}$, shared by the two components, one of which is driving the molecular outflow that has a dynamical timescale of less than a few thousand years. The jets of the two individual components are not aligned. Submillimeter observations show that the region lacks the typical hot-core chemistry.

Conclusions. We find two protostellar objects, whose associated circumstellar and parent core masses are high enough to suggest that they may evolve into intermediate-mass stars. This is corroborated by their association with a very massive and energetic CO outflow, suggesting high protostellar accretion rates. The short dynamical timescale of the outflow, the pristine chemical composition of the cloud core and absence of hot core tracers, the absence of detectable radio continuum emission, and the very low protostellar luminosity argue for an extremely early evolutionary stage.
\end{abstract}

Key words. stars: formation - ISM: jets and outflows

\section{Introduction}

In the investigation of the earliest stages of intermediate- and high-mass star formation, the relative importance of formation processes as different as disk accretion and coalescence is still an open question (for a recent review, see e.g. Beuther et al. 2007). Detailed multi-wavelength studies are needed to illuminate the often confusing observational picture.

Since high-mass protostars are less ubiquitous than their low-mass counterparts, they are on average more distant. The resulting low linear resolution often leads to an oversimplified picture. This is well illustrated by the study of Beuther et al. (2002a), which reported a case where a single bipolar molecular outflow discovered previously with single-dish radio telescopes in a massive-star-forming (MSF) region was resolved into a multiple outflow system in interferometric data (see also Beuther et al. 2003). Davis et al. (2004) analyzed near-infrared data of two collimated jets in a similar MSF region. They conclude that these jets are very similar to their low-mass counterparts.

We report the results of a comprehensive multi-wavelength follow-up study of a candidate massive protostellar source that was discovered close to IRAS 07029-1215 during a millimeter continuum survey of the surroundings of luminous IRAS sources in the outer galaxy (Klein et al. 2005). UYSO 1 is a deeply embedded, very young source at a distance of only $1 \mathrm{kpc}$, powering a high-velocity bipolar $\mathrm{CO}$ outflow; Forbrich et al. (2004) (Paper I) derived the mass of the UYSO 1 cloud core from the $\mathrm{CO}(3-2)$ line emission to be $44 M_{\odot}$, inferring the hydrogen column density from the integrated $\mathrm{CO}$ emission, while the mass derived from optically thin dust continuum emission $(\lambda=850 \mu \mathrm{m})$, tracing high column densities, is $15 M_{\odot}$. The mass of the outflow was estimated to be $5.4 M_{\odot}$. For details on these earlier mass estimates as well as for a discussion of the distance, we refer to Paper I. Compared to other massive molecular outflows, this mass is at the lower end of the range reported 
by Beuther et al. (2002b), but most of their sources are much more distant, at an average distance of $4.7 \pm 3.4 \mathrm{kpc}$. No plausible driving source could be identified in IRAS and MSX midand far-infrared data. In single-dish observations, Beuther et al. (2008) detected $\mathrm{C}_{2} \mathrm{H}$ submillimeter emission towards UYSO 1, a transition that may be a tracer of the earliest stages of (massive) star formation.

The aim of our follow-up observations of UYSO 1 was to study the region at higher angular resolution and to search for the driving source of the enormous CO outflow. For these purposes, we conducted observations in the infrared regime as well as in the millimetric to centimetric wavelength ranges. We note that for very early evolutionary stages, outflow mass entrainment rates and column densities are more conclusive in determining whether a massive star forms than a mass determination since material is still rapidly accreted. In Sect. 2, we describe the variety of observations carried out before presenting the results in Sect. 3. We discuss our findings in Sect. 4 and conclude in Sect. 5.

\section{Observations and data analysis}

Infrared observations. In the near infrared, observations were first performed with the $3.5 \mathrm{~m}$ telescope on Calar Alto, Spain, using the OmegaPrime camera on March 5, 2004, both in $K^{\prime}$ broadband and $\mathrm{H}_{2} \mathrm{~S}(1)$ narrowband (NB2122). After a tentative discovery of two jets, intersecting at the position of UYSO 1, follow-up observations were conducted on several occasions between January 10 and March 1, 2005, with the Infrared Spectrometer and Array Camera (ISAAC) at the Very Large Telescope (VLT) of the European Southern Observatory (ESO). These observations included $K_{\mathrm{s}}, \mathrm{NB} 2.09, \mathrm{NB} 2.13$, as well as $L$ band imaging. In January and February 2006, UYSO 1 was observed with the mid-infrared instrument VISIR at the VLT in the $P A H 2$ filter centered on $\lambda=11.3 \mu \mathrm{m}$, with an estimated $3 \sigma$ point-source sensitivity of $9 \mathrm{mJy}$ (using the VISIR Exposure Time Estimator v3.2.1). The Calar Alto data were reduced using IRAF while we used the ESO Eclipse 5.0 software for the VLT data.

We used the Multiband Imaging Photometer for Spitzer (MIPS, Rieke et al. 2004) onboard the Spitzer Space Telescope to obtain maps at $24 \mu \mathrm{m}$ and $70 \mu \mathrm{m}$ as well as a low-resolution spectrum from $52 \mu \mathrm{m}$ to $105 \mu \mathrm{m}$ in the SED mode. The observations were executed on November, 11 and 12, 2005. The imaging data presented here are post-BCD maps processed with version S16.1 of the pipeline. Inspection of the post-BCD data products and manual reduction with the mopex software package showed that the pipeline reduction of the data is reliable in our case. The widespread extended emission made estimating the background difficult. Some striping along the detector columns remain in the $70 \mu \mathrm{m}$ maps. For display, the stripes were reduced by smoothing with a three-pixel median filter aligned perpendicularly to them. Measurements, however, were done on the unsmoothed maps. The MIPS-SED data were reprocessed with mopex, starting with the BCD products created by the pipeline (S16.1.1) to create the spectra along the slit.

Millimeter and submillimeter observations. Since the discovery observation of the CO outflow, two considerably larger and fully sampled $\mathrm{CO}(3-2)$ maps of the region were taken on June 4 and 6, 2005, with the facility receiver B3 at the JamesClerk-Maxwell Telescope (JCMT). In December 2003, a map of UYSO 1 and its surroundings in $\mathrm{CO}(2-1)$ was taken with the
Heterodyne Receiver Array HERA at the IRAM 30 m telescope. However, since the $\mathrm{CO}(3-2)$ data cover a larger area at a better signal-to-noise ratio, we focus our analysis on these data. In July $2005, \mathrm{~N}_{2} \mathrm{H}^{+}(1-0), \mathrm{N}_{2} \mathrm{D}^{+}(1-0), \mathrm{HCO}^{+}(3-2)$ and $\mathrm{DCO}^{+}(2-1)$ were observed towards UYSO 1 with the IRAM 30 m telescope and its facility receivers in frequency switching mode.

After first, very short observations with the IRAM Plateau de Bure Interferometer $(\mathrm{PdBI})$ in its low-angular resolution D configuration in September/October 2003, UYSO 1 was reobserved in the newly extended high-resolution A configuration in February 2006. While the synthesized beam size in the former observations was $13^{\prime \prime} 9 \times 5^{\prime \prime}$. 9 for the $3 \mathrm{~mm}$ continuum, this improved to $2.2^{\prime \prime} \times 0.6^{\prime \prime}$ in the latter $\left(0.85^{\prime \prime} \times 0.23^{\prime \prime}\right.$ at $\left.1 \mathrm{~mm}\right)$. Besides the continuum, the $\mathrm{CS}(2-1)$ and $\mathrm{CO}(2-1)$ transitions were observed. In order to be able to account for short-spacings in the $u v$ plane, the source was previously mapped in both transitions with the IRAM 30 m telescope.

In November 2005, UYSO 1 was observed with the Caltech Submillimeter Observatory (CSO) on four occasions (Nov. 17/18, Nov. 19, Nov. 20, Nov. 22), looking for several submillimeter transitions in the $345 \mathrm{GHz}$ and $220 \mathrm{GHz}$ ranges. The beam sizes ${ }^{1}(F W H M)$ of the CSO are $\sim 22^{\prime \prime}$ and $\sim 31^{\prime \prime}$ in the $345 \mathrm{GHz}$ and $220 \mathrm{GHz}$ bands, respectively. In the same month, the source was also observed with the Atacama Pathfinder Experiment (APEX) telescope (Güsten et al. 2006) to obtain a better $\mathrm{CO}(3-2)$ spectrum at the source position. The $F W H M$ beam size ${ }^{2}$ of the APEX telescope at $345 \mathrm{GHz}$ is $\sim 18^{\prime \prime}$. All data from the $30 \mathrm{~m}$, APEX, and the PdBI were reduced using the GILDAS software developed by IRAM and Observatoire de Grenoble.

Centimeter radio observations. In November 2003, $\mathrm{H}_{2} \mathrm{O}$ maser emission towards UYSO 1 was discovered with the Effelsberg $100 \mathrm{~m}$ radio telescope. The discovery was confirmed with the same telescope in March 2004. At the same time, as well as in January 2004, the source was searched for $\mathrm{NH}_{3}(1,1)$ emission with the Effelsberg $100 \mathrm{~m}$ telescope. On April 13, 2005, it was searched for signs of $\mathrm{CH}_{3} \mathrm{OH}$ maser emission, using the Torun $32 \mathrm{~m}$ radio telescope.

In March 2006, high-resolution radio observations were carried out with the NRAO Very Large Array (VLA) in A configuration. Both the $8.4 \mathrm{GHz}$ radio continuum emission as well as the newly discovered $\mathrm{H}_{2} \mathrm{O}$ maser emission were studied. The Effelsberg data were reduced using the IRAM GILDAS software, and for the VLA data, we used the NRAO AIPS software.

\section{Results}

Based on the new multi-wavelength data, we report the discovery of two collimated jets that appear to be connected to two previously unresolved protostars at the location of UYSO 1. In spite of deep searches, we did not detect any infrared counterparts of these protostars. Towards one of the two protostars, water maser emission was discovered. Observations of molecular transitions reveal that UYSO 1 does not show typical hot-core chemistry. In the following, we present these results in more detail, differentiating large-scale structure (the clump in which UYSO 1 is embedded, the outflow, and the jets) and small-scale structure (the protostars UYSO 1a and UYSO 1b) before discussing the

\footnotetext{
${ }^{1}$ http://www. submm. caltech.edu/cso/receivers/beams. html

${ }^{2}$ http://www. apex-telescope.org/telescope/
} 
Table 1. Table of relevant positions ${ }^{a}$ (Epoch 2000).

\begin{tabular}{lll}
\hline \hline Name & \multicolumn{1}{c}{ RA (h:m:s) } & \multicolumn{1}{c}{ Dec $\left({ }^{\circ}::^{\prime \prime}\right)$} \\
\hline UYSO 1a & $07: 05: 10.940(1)$ & $-12: 19: 00.64(3)$ \\
UYSO 1b & $07: 05: 10.811(3)$ & $-12: 18: 56.84(9)$ \\
$\mathrm{H}_{2} \mathrm{O}$ maser & $070510.8105(1)$ & $-12: 18: 56.807(3)$ \\
1st 70 $\mu$ m peak & $07: 05: 10.96$ & $-12: 19: 09.9$ \\
2nd 70 $\mu$ m peak & $07: 05: 11.09$ & $-12: 19: 33.1$ \\
24 $\mu$ m peak & $07: 05: 11.56$ & $-12: 19: 19.4$ \\
IRAS 07029-1215 & $07: 05: 16.9$ & $-12: 20: 02$ \\
\hline
\end{tabular}

${ }^{a}$ Numbers in brackets denote the uncertainty in the last digit.

spectral energy distribution (SED). Relevant positions are listed in Table 1.

\subsection{Large-scale structure}

Infrared observations. The initial discovery of two collimated jets, obtained with the Calar Alto $3.5 \mathrm{~m}$ telescope (Fig. 1), was clearly confirmed by the VLT observations (Fig. 2). The larger field of view of the Calar Alto narrowband image additionally shows $\mathrm{H}_{2} \mathrm{~S}(1)$ emission in the outer parts of the $\mathrm{H}$ II region Sh2-297 which is powered by HD 53623. The two jets intersect with an angle of $\sim 75^{\circ}$ at about the submillimeter continuum position of UYSO 1, a first indication that UYSO 1 may harbor more than one source. The larger north-south jet has an apparent size of $1.3^{\prime}$, corresponding to $0.4 \mathrm{pc}$ at a distance of $1 \mathrm{kpc}$. It also has multiple bow shocks, best seen in the continuumsubtracted image in Fig. 3. This jet deviates considerably from a straight line in its northern lobe, possibly a sign of interaction with the surrounding medium. The smaller jet, terminating in a bright, very compact bow shock at its eastern end, is at least $0.2 \mathrm{pc}$ long (it might be even longer, as a barely visible chain of features extends well beyond the bright section of its western lobe). Both jets have a knotty structure. A quantitative estimate of the inclination angles of the two jets with respect to the line of sight is difficult. However, the fact that we clearly see both lobes of both jets in spite of the surrounding material suggests that they lie close to the plane of the sky. The narrowband luminosities of the two jets, as determined from the $2.12 \mu \mathrm{m}$ data, are $L_{2.12 \mu \mathrm{m}}=0.02 L_{\odot}$ for the larger, north-south jet and $L_{2.12 \mu \mathrm{m}}=0.0007 L_{\odot}$ for the smaller, east-west jet. The luminosity of the larger flow is at the upper end of the range of values found in a survey of Orion A (Stanke et al. 2002), a first suggestion that the driving source is at least of intermediate mass.

Another prominent feature is the ridge of strong $\mathrm{H}_{2}$ emission east of the core, extending from the north to the south, also visible in the Calar Alto image. This ridge appears to be the edge of the molecular clump where UYSO 1 is embedded in since also the $\mathrm{CO}$ and millimeter continuum emission drop dramatically in that region. The emission ridge indicates where the $\mathrm{H}$ II region is interfacing the molecular cloud. Whether the $\mathrm{H}_{2}$ emission is excited by shock fronts in the ionisation front or by UV pumping in the photon-dominated region (PDR) between the $\mathrm{H}$ II region and the molecular cloud, we cannot decide, although a PDR appears more likely (see the discussion of oxygen fine structure lines in Sect. 3.3). This would require the comparison of the relative intensities of several $\mathrm{H}_{2}$ lines (e.g., Hollenbach \& Shull 1977), data that are not available at present. The $\mathrm{H}_{2}$ emission in jets is usually shock-excited.

Neither $24 \mu \mathrm{m}$ nor $70 \mu \mathrm{m}$ mid-infrared sources were found to be in direct relation to the millimetre continuum peak (Fig. 4), even though the $70 \mu \mathrm{m}$ observations may show some emission

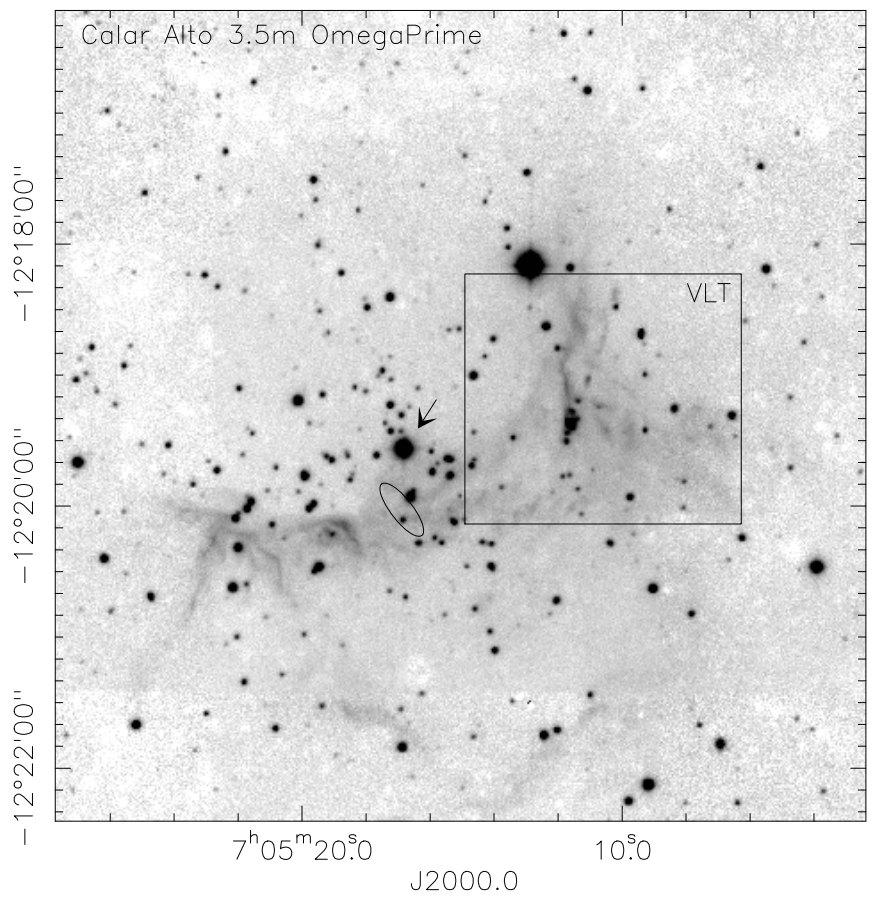

Fig. 1. $\mathrm{H}_{2} \mathrm{~S}(1)$ emission, as observed with OmegaPrime at the Calar Alto $3.5 \mathrm{~m}$ telescope (filter NB2122), in logarithmic scaling. The box denotes the close-up VLT view in Fig. 2. The B star HD 53623 is marked by an arrow and the location of IRAS 07029-1215 is marked by its uncertainty ellipse.

at that position (see discussion of the SED in Sect. 3.3). Instead, the $24 \mu \mathrm{m}$ emission closely follows the $\mathrm{H}_{2} \mathrm{~S}(1)$ emission ridge as the PDR is also heating the dust at the surface of the clump. The $70 \mu \mathrm{m}$ emission also starts at this rim, but extends further into the cloud as it traces colder material deeper in the cloud. The emission peak in the $70 \mu \mathrm{m}$ image is separated by $13^{\prime \prime}$ to the north-west from the $24 \mu \mathrm{m}$ peak. It is still another $11^{\prime \prime}$ away from the millimeter sources. In Sect. 3.3, we discuss the possibility of UYSO 1 being swamped by surrounding extended emission and try to determine its potential contribution to the FIR emission.

(Sub-)millimeter and centimeter radio observations. The new $\mathrm{CO}(3-2)$ single-dish map, shown in Fig. 2, corroborates the results presented in Paper I. There is a strong gradient in emission towards the $\mathrm{H}$ II region east of the position of UYSO 1. The APEX CO(3-2) spectrum taken at the position of UYSO 1 shows the line wings most clearly (Fig. 5). The outflow is prominent in both the $\mathrm{CO}(3-2)$ and the $\mathrm{CO}(2-1)$ maps. Within the uncertainties, the molecular outflow coincides with the larger of the two NIR jets. Thus, in the single-dish CO maps, one massive outflow already discussed in Paper I is detected, but the new, larger $\mathrm{CO}(3-2)$ map additionally shows an indication of an east-west outflow, at least at its western end. Possibly, the millimeter outflow related to the smaller jet is weaker than the large previously known outflow and as such difficult to detect when both systems are superimposed.

From the $\mathrm{CO}(3-2)$ map, the mass of the outflow can be estimated in direct comparison to Paper I, following the procedure outlined there as well as in Henning et al. (2000), using the proportionality of the integrated $\mathrm{CO}$ main-beam temperature and $\mathrm{H}_{2}$ column density. The new, larger map has a slightly smaller SNR than the data used in Paper I, affecting the line wings. For the entire line profile, the $40 \%$ intensity contour (which is not 


\section{arcsec}

$30-10-60$

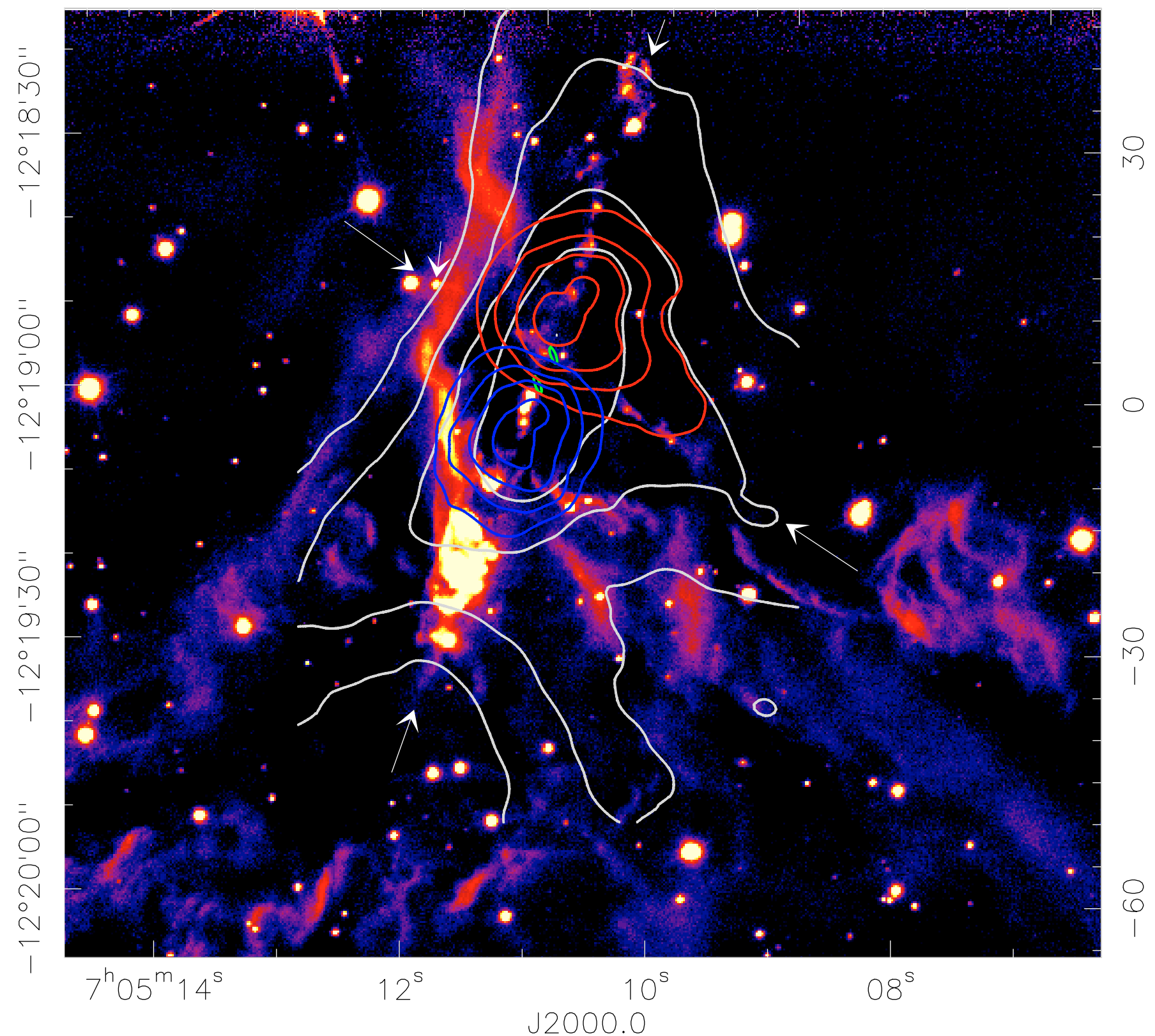

Fig. 2. $\mathrm{H}_{2} \mathrm{~S}(1)$ emission (NB2.13) in logarithmic scaling, overlaid with the JCMT CO(3-2) observations - total line $10 \%$ to $90 \%$ in $10 \%$ steps, line wings $30 \%, 50 \%, 70 \%$ and $90 \%$ - as well as the two continuum sources seen with the PdBI (green ellipses indicating the FWHM synthesized beam). Four arrows indicate the locations of the jets, and an additional small arrow indicates the eastern bow shock of the smaller jet.

entirely within the map) traces $33 M_{\odot}$; the red- and blueshifted line wings trace $2 M_{\odot}$ and $1 M_{\odot}$, respectively (down to the $10 \%$ contour). These numbers are slightly lower than those derived from the deeper data in Paper I, where the cloud mass was estimated to be $40 M_{\odot}$. While it is difficult to give quantitative uncertainties for these mass estimates, we note that the masses are uncertain by at least a factor of a few, i.e., they are orderof-magnitude estimates. While for the extent and the maximum velocities of the outflows, the original JCMT data in the outflow lobes are still the best due to their SNR, we note that our deeper APEX pointing on UYSO 1 shows line wings that are comparable to what was previously only detectable in the outflow lobes. The outflow velocity $v_{\text {proj }}=30 \mathrm{~km} \mathrm{~s}^{-1}$ from Paper I compares to $v_{\text {proj }}=26.5 \mathrm{~km} \mathrm{~s}^{-1}$ derived at the position of UYSO 1 (Fig. 5, compare to Fig. 4, in Paper I). In the new, large $\mathrm{CO}(3-2)$ map, the $10 \%$ contours of the line wings indicate a total size of the molecular outflow of 52 ", or $0.25 \mathrm{pc}$. Compared to Paper I, the inferred size of the outflow is basically unchanged. Assuming that we have an edge-on disk geometry rather than a pole-on view (as is suggested by the clear detection of both near-infrared jet lobes) allows us to better constrain the outflow and its mass entrainment rate. An inclination angle of $80^{\circ}$ with respect to the line of sight yields an outflow dynamical timescale $\left(t_{d}(i)=R_{\text {out }}(i) / v_{\text {out }}(i)\right)$ of only a few hundred years 

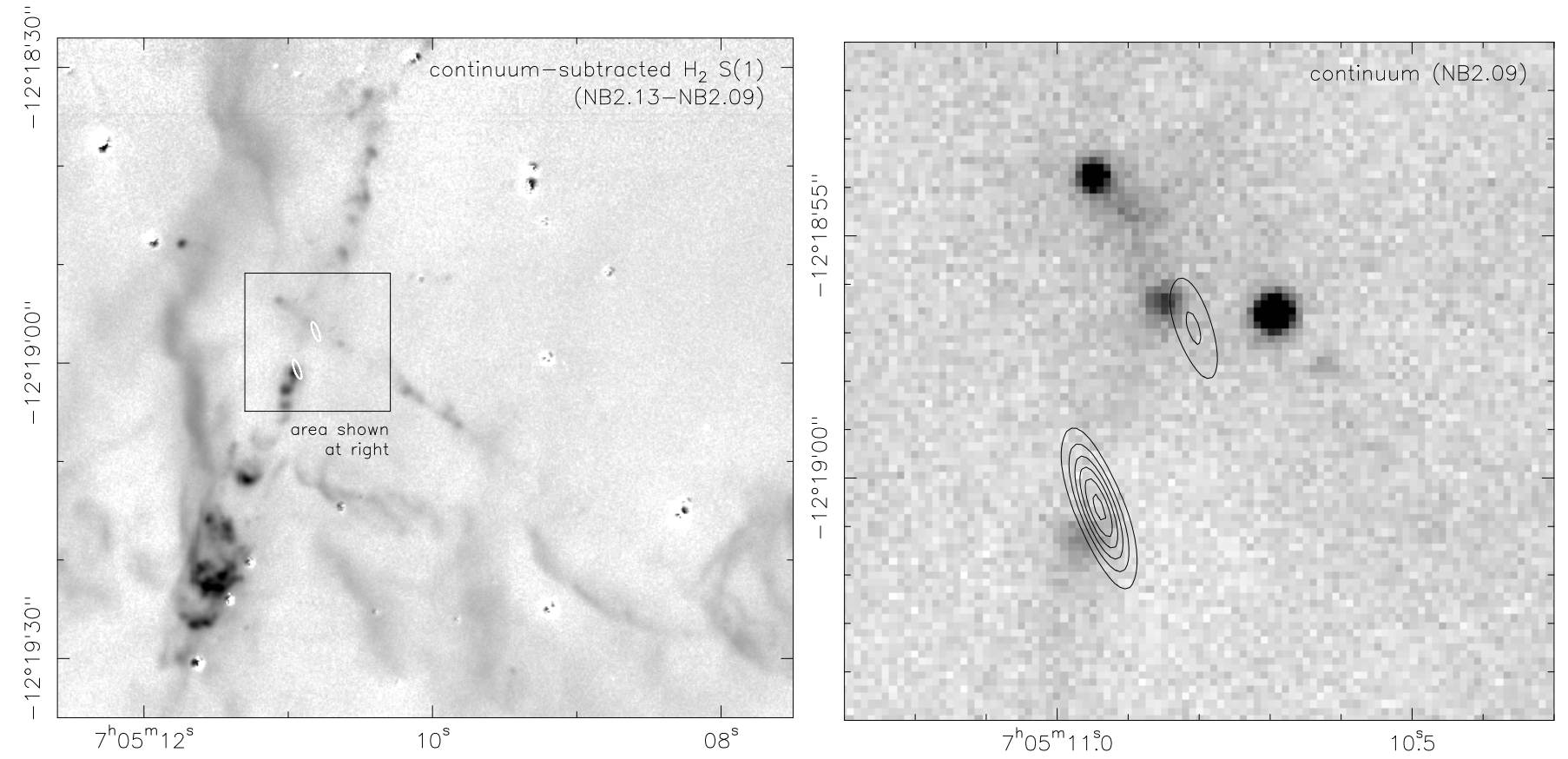

Fig. 3. Left: continuum-subtracted $\mathrm{H}_{2} \mathrm{~S}(1)$ emission (NB2.13-NB2.09) in logarithmic scaling. The subtraction of the brightest stars left a few artefacts. The box indicates the field of view of the right panel; ellipses indicate the positions of the two millimeter continuum sources as in Fig. 2. Right: NIR-continuum-only (NB2.09) close-up view of the central area in linear scaling with the $3 \mathrm{~mm}$ PdBI continuum data shown as contours in steps of $1 \mathrm{mJy}$.

and an outflow mass entrainment rate of $\dot{M}=4 \times 10^{-3} M_{\odot} \mathrm{yr}^{-1}$. Even for an inclination of $i=57.3^{\circ}$ (see Paper I), the dynamical timescale is still only 3000 years with a mass entrainment rate of $\dot{M}=1 \times 10^{-3} M_{\odot} \mathrm{yr}^{-1}$. We can conservatively constrain the dynamical timescale to less than $10^{4} \mathrm{yr}$ except for very small inclination angles $\left(<25^{\circ}\right)$ and the mass entrainment rate correspondingly to $>3 \times 10^{-4} M_{\odot} \mathrm{yr}^{-1}$ : as discussed above, also the NIR observations of the jet lobes suggest large inclination angles with respect to the line of sight. Also, in a pole-on configuration with small inclination angles, we should see the driving sources in the NIR/MIR when looking into the outflow cavities, which is not the case. Based on these outflow properties, we estimate and discuss the accretion luminosity in Sect. 4.

New molecular line observations beyond those in Paper I are summarized in Table 2. To better trace the overall mass, we also studied UYSO 1 in ${ }^{13} \mathrm{CO}(2-1)$. Following Scoville et al. (1986), the emission peak at the position of UYSO 1 corresponds to a mass of $8 M_{\odot}$ in the CSO beam size of $\sim 33^{\prime \prime}(F W H M)$. Interestingly, neither $\mathrm{NH}_{3}$ nor $\mathrm{N}_{2} \mathrm{H}^{+}(1-0)$ emission was found towards the source, with upper limits of $<0.15 \mathrm{~K}$ and $<0.1 \mathrm{~K}$, respectively. $\mathrm{HCO}^{+}(3-2)$ and $\mathrm{DCO}^{+}(2-1)$ were detected. The $\mathrm{HCO}^{+}(3-2)$ line may consist of several velocity components. Several submillimeter molecular lines that we searched for using the CSO were not detected (see footnote in Table 2). These results indicate that UYSO 1, with only very few detectable molecular transition lines, does not show typical hot-core chemistry.

Based on the upper limit of $\mathrm{N}_{2} \mathrm{H}^{+}(1-0)$ emission, we can estimate corresponding upper limits for the column density and the abundance. For the column density, $N_{\mathrm{N}_{2} \mathrm{H}^{+}} \approx 8 \times 10^{11} \Delta v T_{R} \mathrm{~cm}^{-2}$ (Benson et al. 1998), we derive an upper limit of $N_{\mathrm{N}_{2} \mathrm{H}^{+}} \approx 1.6 \times$ $10^{11} \mathrm{~cm}^{-2}$, assuming a line width of $1 \mathrm{~km} \mathrm{~s}^{-1}$. An upper limit for the abundance follows when relating this to the hydrogen column density derived by Klein et al. (2005) from the submillimeter radio data at $850 \mu \mathrm{m}, N_{\mathrm{H}}=5.8 \times 10^{22} \mathrm{~cm}^{-2}$ (for $T=20 \mathrm{~K}$ ). The result, an abundance of only $N_{\mathrm{N}_{2} \mathrm{H}^{+}} / N_{\mathrm{H}} \leq 2.6 \times 10^{-12}$, is surprisingly low compared to values of $\approx 10^{-10}$ found in starless cores by Tafalla et al. (2004).

\subsection{Small-scale structure}

Millimeter observations. The millimeter continuum data from the two PdBI observations are ideal to study the small-scale dust continuum emission. In the low-resolution (D configuration) data, only a single source is detected at $3 \mathrm{~mm}$. In the highresolution data (extended A configuration), this source is resolved into two protostars, UYSO 1a and UYSO 1b (Table 1); both sources remain unresolved. At $1 \mathrm{~mm}$, only the brighter southern source, UYSO 1a, is clearly detected due to limited sensitivity. Results of the two $3 \mathrm{~mm}$ continuum datasets are shown in Fig. 6.

We follow Beuther et al. $(2003,2005)$ in determining the mass from the $3 \mathrm{~mm}$ continuum data, as traced by the optically thin dust emission of the protostellar envelopes. We assume a temperature of $T=45 \mathrm{~K}$ (Paper I) while leaving the gas-to-dust ratio (100:1) and other quantities the same as in Beuther et al. (2003) who estimate the results to be accurate within a factor of five. The single source detected in the low-resolution data corresponds to a gas mass of $9.5 M_{\odot}$. In the high-resolution data, two continuum sources are detected in the $3 \mathrm{~mm}$ band. These are at roughly the positions of two faint near-infrared continuum sources, but do not fit exactly (see discussion of the NIR observations below). Both objects appear to be intermediatemass protostars with gas masses of $3.5 M_{\odot}$ for the more massive component, UYSO $1 \mathrm{a}$, and $1.2 M_{\odot}$ for the second component, UYSO $1 \mathrm{~b}$. The linear separation between the two sources is 4.' 17, or 4200 AU. The hydrogen column densities corresponding to the derived gas masses are $N_{\mathrm{H}}=6.2 \times 10^{24} \mathrm{~cm}^{-2}$ and $N_{\mathrm{H}}=2.2 \times 10^{24} \mathrm{~cm}^{-2}$ for UYSO $1 \mathrm{a}$ and UYSO $1 \mathrm{~b}$, respectively. As a crude approximation, we convert these to visual extinction according to the empirical relation $N_{\mathrm{H}}\left[\mathrm{cm}^{-2}\right] \approx$ $2 \times 10^{21} \times A_{\mathrm{V}}[\mathrm{mag}]($ Ryter 1996; Vuong et al. 2003), resulting in 


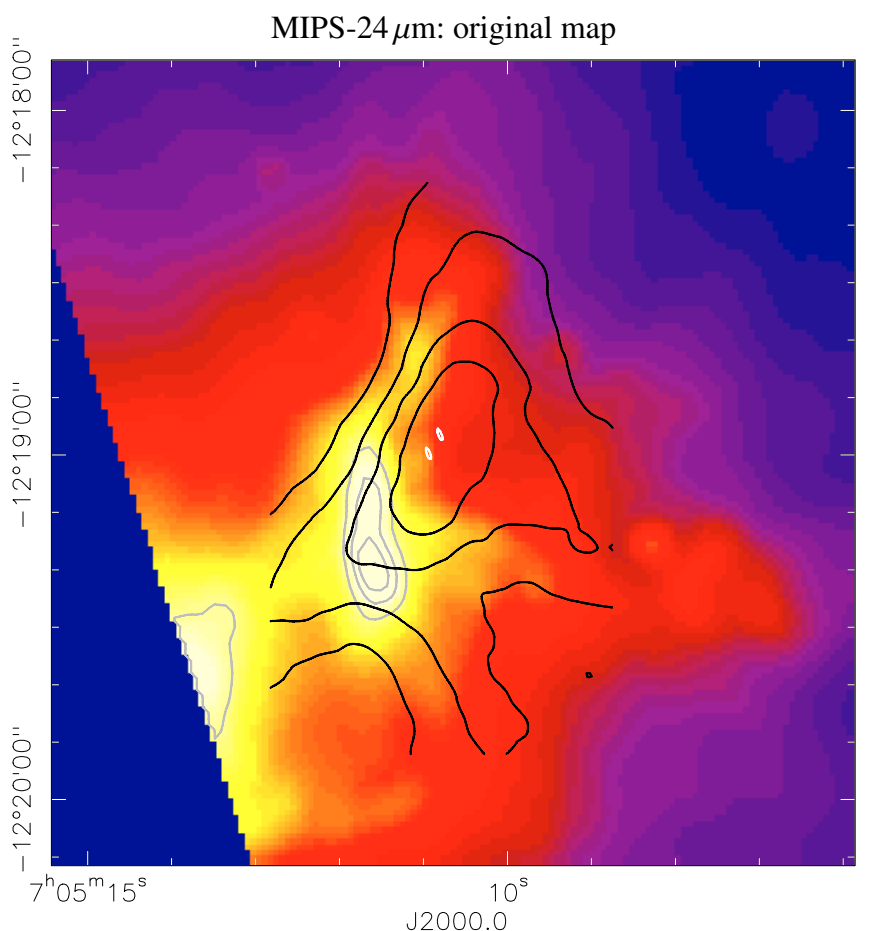

MIPS-24 $\mu \mathrm{m}$ : map with extended emission subtracted

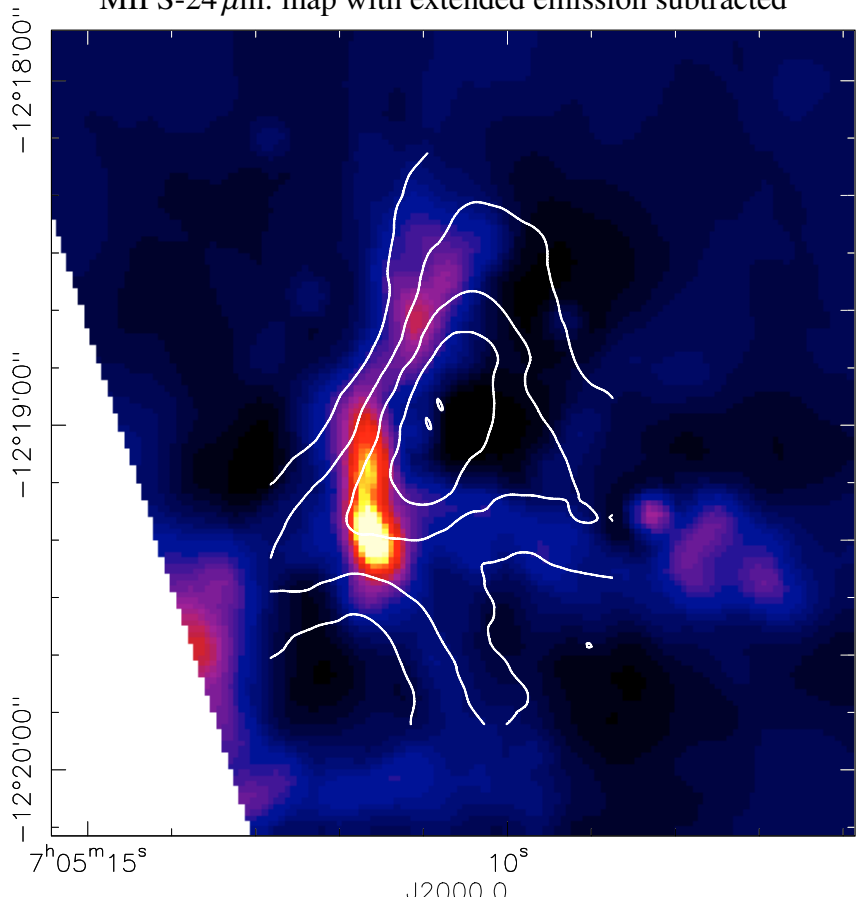

MIPS-70 $\mu \mathrm{m}$ : original map

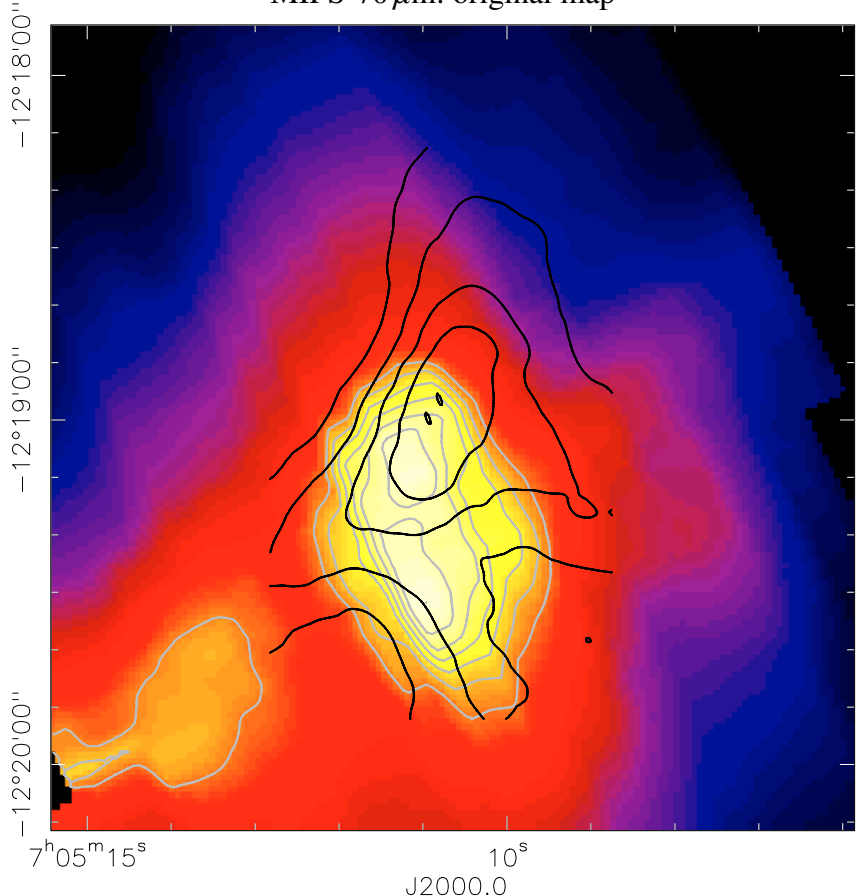

MIPS-70 $\mu \mathrm{m}$ : map with extended emission subtracted

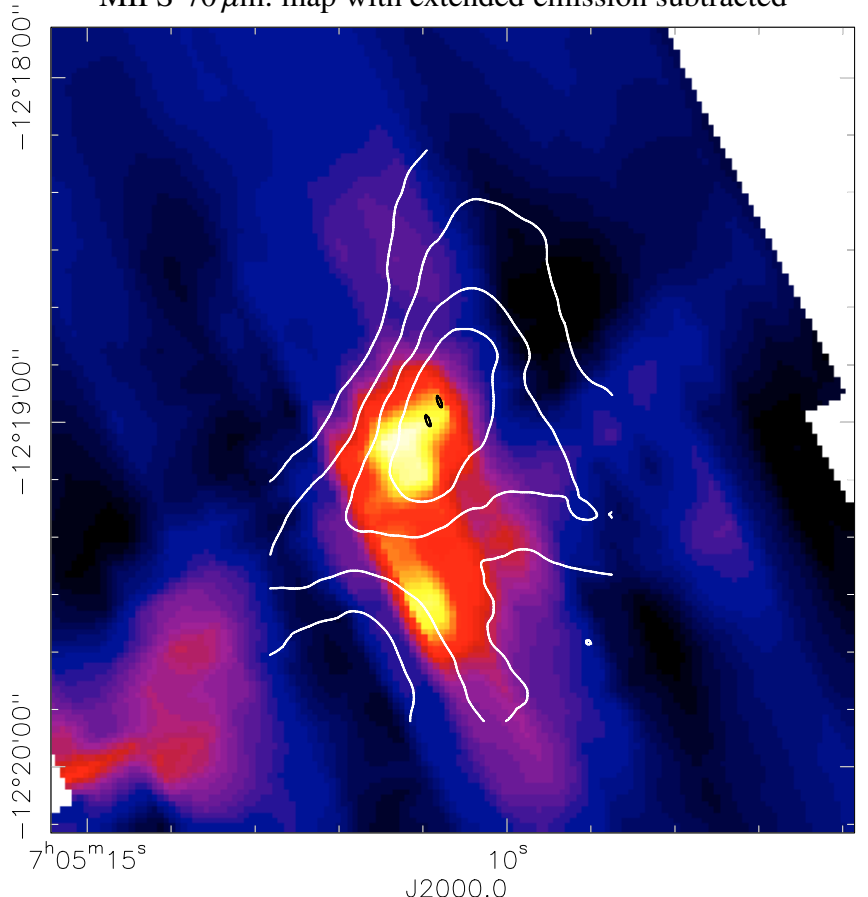

Fig. 4. MIPS $24 \mu \mathrm{m}$ (left) and $70 \mu \mathrm{m}$ (right) data. Upper panel: original maps, in logarithmic scaling. Contours of the data (grey) and of the $\mathrm{CO}(3-2)$ emission (black) are displayed as reference. Lower panel: maps with extended emission filtered out (see text), in linear scaling. Contours of the $\mathrm{CO}(3-2)$ emission are displayed as reference. The color scale is different from the one used in the upper panel. In all plots, the locations of UYSO 1a and UYSO 1b are marked with small ellipses, as in Fig. 2.

visual extinctions of $A_{V}>1000$ mag. We note that the derived column densities are well above typical values for low-mass protostars (e.g., Motte \& André 2001) and also above the minimum column density of $3 \times 10^{23} \mathrm{~cm}^{-2}$ (or $1 \mathrm{~g} \mathrm{~cm}^{-2}$ ) that Krumholz \& McKee (2008) derived for the formation of massive stars.

The molecular line data collected with the PdBI suffers from missing flux, and the two extreme configurations are difficult to combine due to virtually no overlap in the $u v$ plane. Thus, we only briefly discuss the $\operatorname{CS}(2-1)$ data here. While in the
D configuration, again only a single source is detected with barely noticable velocity structure, the A configuration data show emission that is largely resolved out.

Centimeter radio observations. $\mathrm{H}_{2} \mathrm{O}$ maser emission, a signpost of low- and high-mass star formation (Henning et al. 1992), was discovered in single-dish observations towards UYSO 1 in two velocity components symmetrically spaced around the 


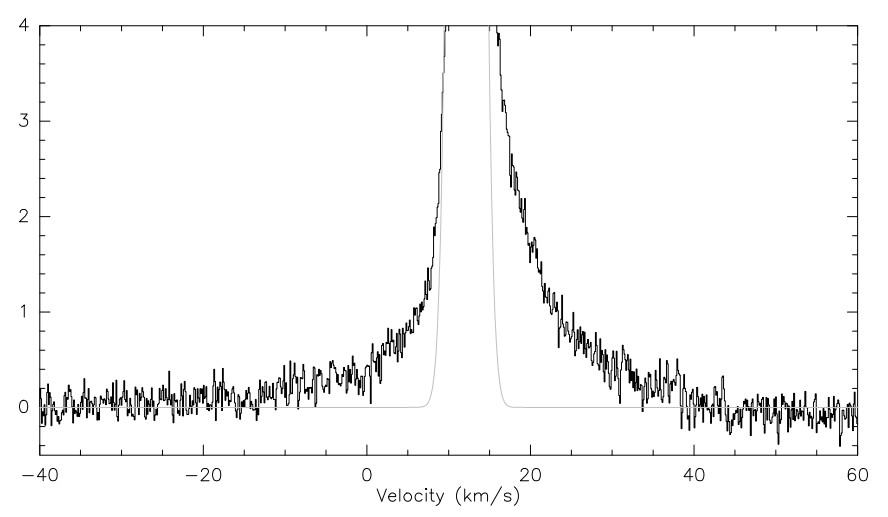

Fig. 5. CO(3-2) spectrum towards UYSO 1, as observed with the APEX telescope, in $T_{a}^{*}[\mathrm{~K}]$. In order to show the line wings, only the lower $10 \%$ part of the line are shown. The grey line shows a Gaussian profile fitted to the entire line.

Table 2. Molecular transition lines observed towards UYSO 1.

\begin{tabular}{lll}
\hline \hline Transition & Telescope & $T_{a}^{*}[\mathrm{~K}]$ \\
\hline $\mathrm{NH}_{3}(1,1)$ & Effelsberg $100 \mathrm{~m}$ & $<0.15 \mathrm{~K}$ \\
$\mathrm{~N}_{2} \mathrm{H}^{+}(1-0)$ & IRAM $30 \mathrm{~m}$ & $<0.1 \mathrm{~K}$ \\
$\mathrm{HCO}^{+}(3-2)$ & IRAM $30 \mathrm{~m}$ & $3.5 \mathrm{~K}$ \\
$\mathrm{DCO}^{+}(2-1)$ & IRAM $30 \mathrm{~m}$ & $0.5 \mathrm{~K}$ \\
${ }^{13} \mathrm{CO}$ & $\mathrm{CSO}^{a}$ & $1.8 \mathrm{~K}$ \\
$\mathrm{HCN}(4-3)$ & $\mathrm{CSO}$ & $0.7 \mathrm{~K}$ \\
$\mathrm{CCH}^{b}$ & $\mathrm{CSO}$ & $<0.4 \mathrm{~K}$ \\
$\mathrm{H}_{2} \mathrm{CO}\left(5_{(1,5)}-4_{(1,4)}\right)$ & $\mathrm{CSO}$ & $0.3 \mathrm{~K}$ \\
\hline
\end{tabular}

a Notable undetected molecules in our $345 \mathrm{GHz}$-range CSO observations: $\mathrm{CH}_{3} \mathrm{CN}, \mathrm{CH}_{3} \mathrm{OH}, \mathrm{SO}, \mathrm{SO}_{2}, \mathrm{HNCO}, \mathrm{HCOOCH}_{3}, \mathrm{HCCCN}$, $\mathrm{CH}_{3} \mathrm{CH}_{2} \mathrm{CN}$, and additionally in the $220 \mathrm{GHz}$ range: $\mathrm{SiO} ;{ }^{b}$ blends at $349.338 \mathrm{GHz}$ and $349.400 \mathrm{GHz}$.

system velocity of $\mathrm{v}_{\mathrm{lsr}}=12 \mathrm{~km} \mathrm{~s}^{-1}$ (Fig. 7). One of the two components has a double-peaked substructure. In high-resolution VLA A-array observations, however, only a single maser spot was found at the position of the north-western millimeter continuum source, UYSO 1b, close in velocity to the brighter component in the Effelsberg maser spectrum. No $\mathrm{CH}_{3} \mathrm{OH}$ maser emission was detected $(<0.3 \mathrm{Jy})$. In the centimeter continuum at wavelengths of $1.3 \mathrm{~cm}$ and $3.5 \mathrm{~cm}$, only $3 \sigma$ upper limits of $5.6 \mathrm{mJy}$ and $0.21 \mathrm{mJy}$ for the flux densities could be determined, respectively. Thus, no detectable $\mathrm{H}$ II region has formed yet.

Near- and mid-infrared observations. In deep NIR imaging, two sources were detected in the NB2.09 continuum and the $L$ band which are close to the positions of the millimeter protostars, but they do not coincide with them (Figs. 2 and 3). The astrometric accuracy of the near-infrared data, refined with positions from the 2MASS catalogue, is estimated to be $<0.2^{\prime \prime}$. Given the enormous visual extinction towards the two protostars (see above), we probably only see scattered light from their vicinities (e.g. Linz et al. 2005; Weigelt et al. 2006). Since UYSO 1a lies in the large north-south jet, and UYSO 1b lies in the east-west jet, the two millimeter sources probably are their driving sources. Then, UYSO 1a would also power the dominant molecular outflow coinciding with its jet while UYSO 1b excites the water maser. Notably, the NB2.09 NIR continuum source just to the south-east of UYSO 1a and the source just to the east of UYSO $1 b$ appear not to be point sources; it is also noteworthy that both appear on the sides of the blueshifted outflow lobes, which would be tilted towards us and thus suffer less extinction.

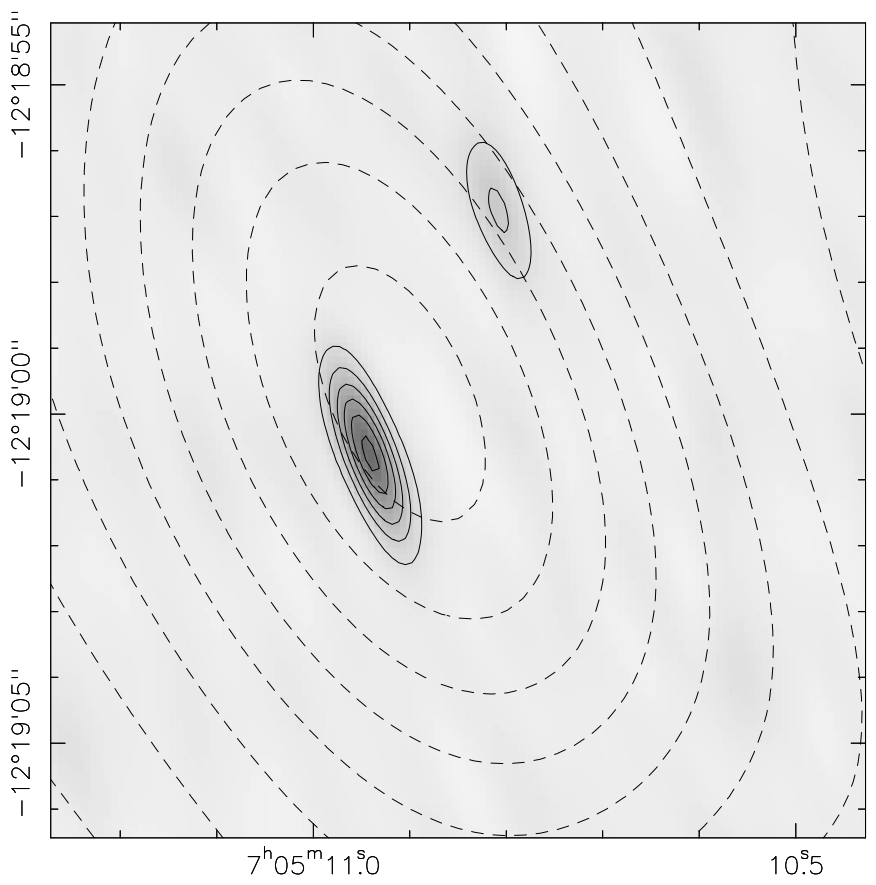

Fig. 6. The two $3 \mathrm{~mm}$ continuum observations using the PdBI, at the same angular scale (greyscale and solid contours: A configuration, contour step: $1 \mathrm{mJy}$; dashed contours: D configuration, contour step: $2 \mathrm{mJy}$ ). The brighter source is UYSO 1a, the fainter one is UYSO $1 \mathrm{~b}$.

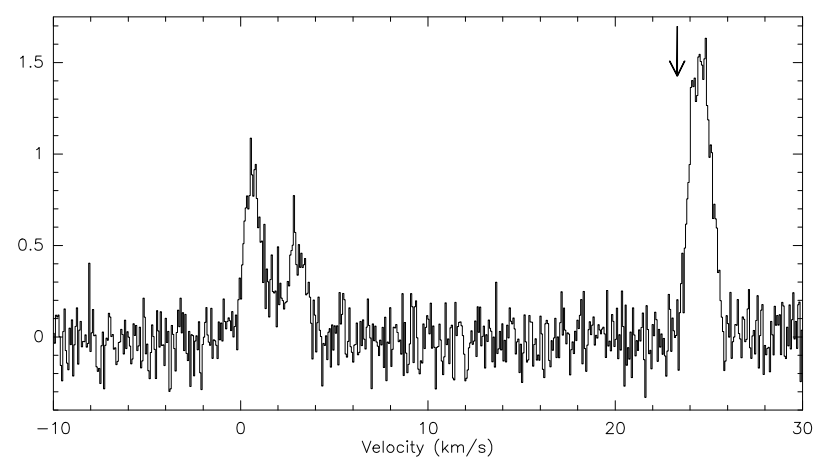

Fig. 7. $\mathrm{H}_{2} \mathrm{O}$ maser emission towards UYSO 1, as observed with the Effelsberg $100 \mathrm{~m}$ telescope, axis units are Jy. The VLA detection of an $\mathrm{H}_{2} \mathrm{O}$ maser in UYSO $1 \mathrm{~b}$ has an unresolved velocity of $23.3 \mathrm{~km} \mathrm{~s}^{-1}$, marked by an arrow, with a peak flux density of $0.92 \mathrm{Jy}$. The system velocity is $v=12 \mathrm{~km} \mathrm{~s}^{-1}$.

In the VISIR observations at $11 \mu \mathrm{m}$, no source was detected at the position of UYSO 1 . As an upper limit, we use the expected $3 \sigma$ point source sensitivity of $9 \mathrm{mJy}$.

\subsection{Spectral energy distribution}

Compared to the spectral energy distribution of UYSO 1 in Paper I which was based on the submillimeter detections and upper limits derived from IRAS data, we now have much more comprehensive information to constrain the combined luminosity of UYSO $1 \mathrm{a}$ and $1 \mathrm{~b}$.

Broad-band Spitzer observations. As noted in Sect. 3.1, UYSO 1 remains undetected in the MIPS scan maps. Bright extended emission from the clump around UYSO 1 is detected but no compact emission peak that could be ascribed to the core 


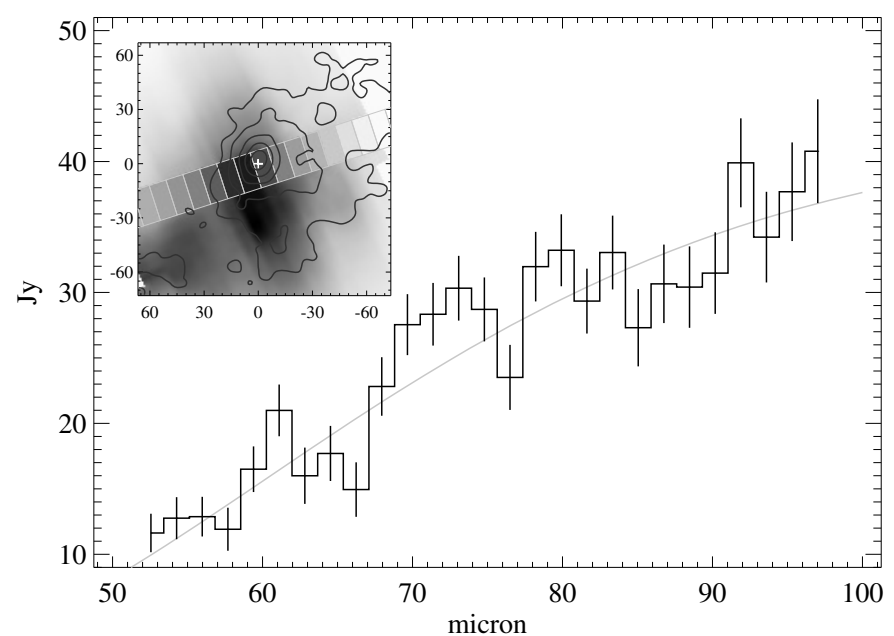

Fig. 8. MIPS spectrum of UYSO 1 with $3 \sigma$ error bars; in grey a $40 \mathrm{~K}$ black body spectrum; inset: Location, size, and integrated intensity of the MIPS slit on $70 \mu \mathrm{m}$-map with $850 \mu \mathrm{m}$-contours

containing UYSO $1 \mathrm{a}$ and $1 \mathrm{~b}$ is seen. The question is how to estimate upper limits for the flux density of UYSO 1 at $24 \mu \mathrm{m}$ and $70 \mu \mathrm{m}$. As a conservative estimate, we perform aperture photometry at the position of UYSO 1, using apertures of $14^{\prime \prime}$ and $32^{\prime \prime}$ in diameter with background annuli from $80^{\prime \prime}$ to $100^{\prime \prime}$ and $78^{\prime \prime}$ to $130^{\prime \prime}$, for 24 and $70 \mu \mathrm{m}$, respectively. These apertures include UYSO 1 but do not cut into the PDR, whereas the background annuli lie beyond the main extended emission. We applied the appropriate aperture and color corrections as described in the MIPS Data Handbook v3.3 and as derived from the theoretical PSF. This method yields conservative upper limits of $0.3 \mathrm{Jy}$ and 70 Jy for UYSO 1 at $24 \mu \mathrm{m}$ and $70 \mu \mathrm{m}$, respectively.

However, these values mainly measure the large-scale emission of the externally heated envelope at the position of UYSO 1. Inspired by unsharp masking, we estimate the extended emission by convolving the maps with a Gaussian profile and then subtracting it to retain the small-scale structure. The FWHM of the Gaussian has been 40". The unsharp-masked maps are displayed in the lower panels of Fig. 4. The small-scale structure is now much more pronounced, but no peak at the position of UYSO 1 becomes apparent. Repeating the aperture-photometry should now result in values much less affected by the extended emission. Virtually no flux is left in the $24 \mu \mathrm{m}$ map at the position of UYSO 1. Only an upper limit of $0.1 \mathrm{Jy}$ can be derived. At $70 \mu \mathrm{m}$, the aperture photometry retains $25 \pm 2 \mathrm{Jy}$ at the position of UYSO 1. This number is a second upper limit since there is no compact emission apparent at this position (although there is a conspicuous extension of the $70 \mu \mathrm{m}$ emission towards the positions of UYSO $1 \mathrm{a}$ and $\mathrm{b}$, possibly indicating that these protostars start to become visible at about this wavelength). There is considerable uncertainty as to how well the extended emission and the cloud background are subtracted.

Low-resolution Spitzer spectroscopy (SED mode). Even though UYSO 1 remains undetected in the MIPS imaging data, the low-resolution spectroscopy can help characterizing the surroundings. The inset of Fig. 8 shows the location, the size, and the pixels of the MIPS slit relative to the $70 \mu \mathrm{m}$ imaging data; the resulting spectrum is shown in the main part of the figure. This spectrum was extracted from the three pixels closest to the position of UYSO 1. The cloud background was removed by subtracting the average of the three pixels east

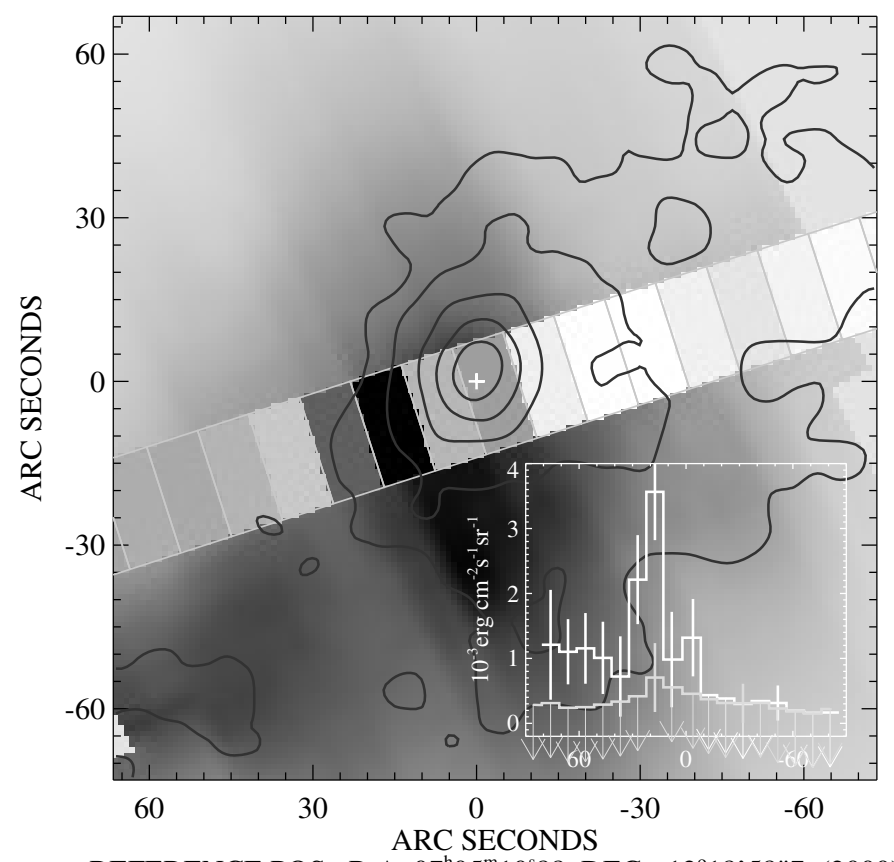

REFERENCE POS. R.A. $07^{\mathrm{h}} 05^{\mathrm{m}} 10^{\mathrm{s}} 88$ DEC $-12^{\mathrm{o}} 18^{\prime} 58.7$ (2000)

Fig. 9. The [OI] and [OIII] line: the intensity in the MIPS slit are the [OI] $63 \mu \mathrm{m}$-line on top of the $70 \mu \mathrm{m}$-map ( $850 \mu \mathrm{m}$ contours). The inset displays again the variation of the [OI] $63 \mu \mathrm{m}$-line across the slit plus the [OIII] $88 \mu \mathrm{m}$-line (grey). Error bars are $3 \sigma$.

and three pixels west of the UYSO 1 spectrum. All the spectra are derived by averaging over three pixels along the slit and applying the proper aperture correction. The $3 \sigma$ error bars are the uncertainties propagated from those reported in the post-BCD products. The spectrum derived for the position of UYSO 1 is compatible with the flux derived from the $70 \mu \mathrm{m}$ map. It does not show any strong features and the slope can be described by a black body of temperature $40 \mathrm{~K}$. Note again that UYSO 1 itself is probably not detected.

Oxygen fine-structure lines. A feature that is detected in the Spitzer SED-mode observations despite its low spectral resolution are oxygen fine structure lines at the eastern rim of the molecular core. Figure 9 again shows the $70 \mu \mathrm{m}$ map with the MIPS slit, this time with the intensities of the [OI] line at $63 \mu \mathrm{m}$. The inset shows the variation of the [OI] line and the [OIII] $(88 \mu \mathrm{m})$ line along the slit. The subtracted continuum was obtained by averaging the two pixels neighboring the linecontaining pixel in wavelength.

We clearly see here a PDR radiating in two important cooling lines on the eastern side of the core where the radiation from IRAS 07029-1215 impacts the molecular cloud. The emission comes only from a small area as atomic oxygen is only present up to optical depths of $A_{V} \approx 10 \mathrm{mag}$, and the PDR must be seen edge on. The [OI] line's intensity of a few $10^{-3} \mathrm{erg} \mathrm{cm}^{-2} \mathrm{~s}^{-1} \mathrm{sr}^{-1}$ is expected for PDRs with densities of $10^{3}$ to $10^{4} \mathrm{~cm}^{-3}$ (Hollenbach \& Tielens 1999).

\section{Discussion}

In view of our previous knowledge on UYSO 1, the main new result that warrants discussion is its newly constrained SED. Also, the nondetections in $\mathrm{N}_{2} \mathrm{H}^{+}$and $\mathrm{NH}_{3}$ are of special interest. 
The non-detection of UYSO 1 at mid-infrared wavelengths is surprising given the earlier estimate of the SED in Paper I. The mid-infrared part of that SED was only weakly constrained by upper limits from IRAS data, clearly contaminated by the nearby bright source IRAS 07029-1215. The previous estimate includes the entire cloud core, heated also externally. The previous luminosity upper limit of $<1900 L_{\odot}$ corresponds to the large-scale emission in the Spitzer maps. Between 24 and $70 \mu \mathrm{m}$, the luminosity of the filtered-out large-scale emission is $\sim 1500 L_{\odot}$.

We can estimate the amount of energy that is intercepted and re-radiated by the PDR neighboring UYSO 1 . The luminosity estimate in Paper I includes any such emission inside the diameter of $80 \%$ encircled energy of IRAS at $100 \mu \mathrm{m}$. Our MIPS data indicate that extended emission is indeed present around UYSO 1 on these scales. The projected distance between UYSO 1 and HD 53623 is $0.46 \mathrm{pc}$. Since the cloud is illuminated from the side, the real distance should not be much different, i.e., $<1 \mathrm{pc}$ (if it protrudes less than $60^{\circ}$ out of the plane of the sky). When seen from HD 53623, the size of the extended emission subtends an angle of $28-60^{\circ}$, or a solid angle of $0.18-0.85 \mathrm{sr}$, assuming that the cloud is at a distance of $1-0.46 \mathrm{pc}$ and appears circular. The remaining uncertainty is the luminosity class of HD 53623 for which spectral types of B1V (Claria 1974) and B1II/III (Houk \& Smith-Moore 1988) are given in the literature. Correspondingly assuming luminosities of $16000 L_{\odot}$ or $39000 L_{\odot}$ (Schmidt-Kaler 1982), the cloud core intercepts and eventually re-radiates $230-2640 L_{\odot}$. With the uncertainties involved, it seems reasonable to assume that the previously estimated upper limit for the luminosity was dominated by externally heated extended emission.

In spite of the new data, the SED is only constrained by upper limits in the infrared regime. Since the newly discovered two components are not resolved in the submillimeter continuum, we use the unresolved PdBI continuum data at $3 \mathrm{~mm}$ (from the D configuration) and do not take into account the high-resolution PdBI data for the SED fit. Assuming an isotropic radiation field, a new modified-blackbody fit (with dust opacities of $\kappa_{v} \propto v^{\beta}$ and $\beta=2$ ) yields a luminosity upper limit of $\sim 50 L_{\odot}$ for both components combined (see Fig. 10), assuming a distance of $1 \mathrm{kpc}$. This may be a severe lower bound if most of the luminosity escapes along the outflow cavities and is not re-processed into infrared radiation.

In spite of the uncertainties involved, it is interesting to compare this luminosity to an estimate of the accretion luminosity. The outflow mass entrainment rate translates into a mass loss rate of the driving jet which in turn translates into the actual accretion rate onto the star. Beuther et al. (2002b) estimate that this actual accretion rate is lower than the outflow mass entrainment rate by a factor of about 6 . The above-mentioned conservative lower limit for the mass entrainment rate thus translates into a continuous accretion rate of $>5 \times 10^{-5} M_{\odot} \mathrm{yr}^{-1}$. A mass entrainment rate of $\dot{M}=4 \times 10^{-3} M_{\odot} \mathrm{yr}^{-1}$ translates into an accretion rate of $7 \times 10^{-4} M_{\odot} \mathrm{yr}^{-1}$. Based on the accretion rate, it is possible to estimate the accretion luminosity, $L_{\mathrm{acc}}=G \cdot M_{\star} \cdot \dot{M}_{\mathrm{acc}} \cdot r_{\star}^{-1}$. Of course, the stellar parameters are barely constrained in this case, and the situation is further complicated by the assumption of isotropic radiation, but we can carry out an order-ofmagnitude check. For a $3 M_{\odot}$ star and a corresponding radius of 5-8 $R_{\odot}$ (Palla \& Stahler 1992; Yorke \& Bodenheimer 2008), the lower limit of the accretion rate corresponds to an accretion luminosity of 600-900 $L_{\odot}$. For comparison, a $10 M_{\odot}$ star with a corresponding radius of $7-12 R_{\odot}$ would have an accretion luminosity of $1300-2200 L_{\odot}$. The estimated accretion luminosity thus appears to be about an order of magnitude larger than

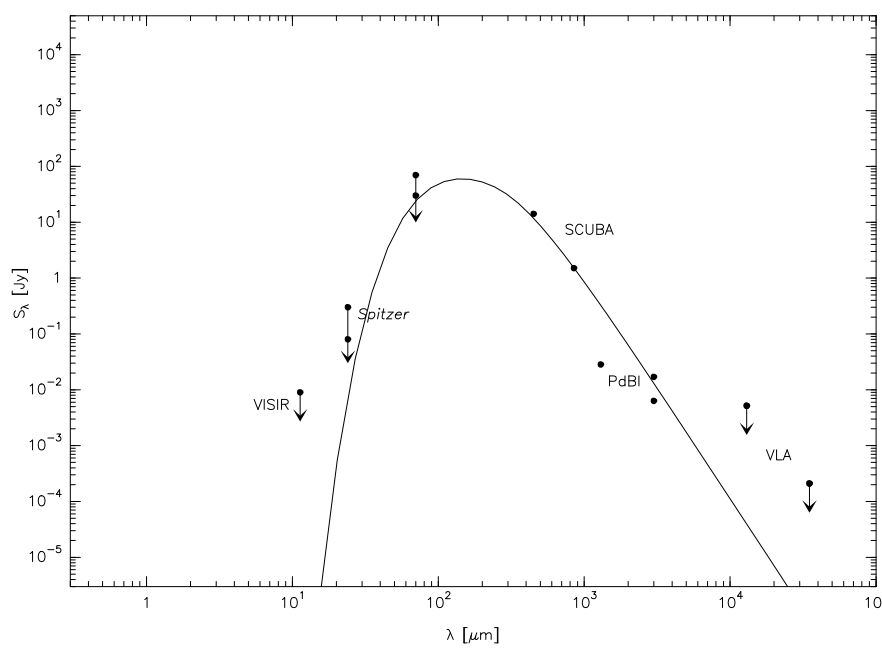

Fig. 10. The SED of the combined two sources that make up UYSO 1. The fit mainly relies on the submillimeter continuum data and the Spitzer upper limits and thus again constitutes only an upper limit in its infrared part. The two data points at $3 \mathrm{~mm}$ correspond to the highresolution and low-resolution PdBI data. Fitting modified-blackbody radiation to this SED yields an upper limit for the estimated total luminosity of $\lesssim 50 L_{\odot}$.

the luminosity upper limit deduced from the SED with the assumption of isotropic radiation. However, we note again that, given the likely presence of accretion disks, the radiation field is highly anisotropic. In particular, such disks would have the highest extinction, poorly determinable in the mid-infrared, in the direction towards the observer. Therefore, and due to the fact that the accretion luminosity is but a simple estimate with uncertain assumptions, the above discussion should be regarded as very tentative.

It remains unclear whether the empirical relation between the mass entrainment rate of the outflow and the luminosity of the central object that was used in Paper I is reliable (Shepherd \& Churchwell 1996; Henning et al. 2000; Beuther et al. 2002b, see also Wu et al. 2005). We note nevertheless that the mass entrainment rate appears to be higher than what would be expected when applying this relation for a driving source with a luminosity of $\sim 50 L_{\odot}$.

UYSO 1 was not observed to show centimetric $\mathrm{NH}_{3}$ emission or millimetric $\mathrm{N}_{2} \mathrm{H}^{+}$emission although we detect $\mathrm{HCO}^{+}(3-$ 2) at the same position. Womack et al. (1991) find a similar effect towards Orion-IRc2 and speculate that $\mathrm{N}_{2} \mathrm{H}^{+}$may be depleted by shocks or winds close to that source. On the other hand, Tafalla \& Santiago (2004) found an extremely young starless core with constant $\mathrm{C}^{18} \mathrm{O}$ and an unusually low $\mathrm{N}_{2} \mathrm{H}^{+}$abundance. The fact that we do not see typical hot-core chemistry towards UYSO 1, combined with the still low luminosity of the two sources suggests that we see very young sources. While it remains unclear how exactly the outflow fits into this picture due to the unknown inclination angle, a sufficiently large inclination angle would explain the extinction towards the central source and lead to a high mass entrainment rate of the outflow.

\section{Conclusions}

We report the detection of two highly collimated jets intersecting close to the position of the previously identified candidate massive protostar UYSO 1. Follow-up high-resolution millimeter radio observations show two continuum sources with masses 
of $3.5 M_{\odot}$ (UYSO 1a) and 1.2 $M_{\odot}$ (UYSO 1b) and high column densities $\left(>10^{24} \mathrm{~cm}^{-2}\right)$ close to the geometric centers of the jets which probably are their powering sources. The projected distance between the two sources is $4200 \mathrm{AU}$. UYSO la appears to power the energetic molecular outflow that was previously discovered towards UYSO 1 . UYSO $1 \mathrm{~b}$ contains a previously unknown $\mathrm{H}_{2} \mathrm{O}$ maser source while a search for $\mathrm{CH}_{3} \mathrm{OH}$ maser emission remained unsuccessful. The two millimeter protostars are not detected in deep near-infrared imaging and remain undetected also at mid- to far-infrared wavelengths. Also, no developing H II regions were found. Submillimeter observations show that the region is rather cold and does not show typical hot-core chemistry. Curiously, not even emission in $\mathrm{NH}_{3}$ or $\mathrm{N}_{2} \mathrm{H}^{+}$was detected. An attempt to constrain the combined SED of the two sources yields an estimated luminosity of roughly $L \lesssim 50 L_{\odot}$, which is about an order of magnitude lower than a tentative estimate of the accretion luminosity. The key to combining these new results into a coherent picture probably lies in the unknown outflow inclination angle with respect to the line of sight. A large inclination angle would lead to configurations in which the circumstellar disk is seen nearly edge-on, obscuring the central object and helping to explain their infrared non-detections. For moderately large inclination angles of about $>25^{\circ}$, where a thick circumstellar disk and a dense envelope would obscure the central object, the molecular outflow powered by UYSO 1a has a dynamical timescale of $<10000$ years with enormous outflow mass entrainment rates of $>\dot{M}=3 \times 10^{-4} M_{\odot} \mathrm{yr}^{-1}$. The luminosity of the corresponding near-infrared jet is comparable to the most luminous jets found in a survey of Orion A. In addition to the peculiar chemistry and the still relatively low luminosity, this indicates a very early evolutionary stage.

Acknowledgements. We wish to thank Sandra Bruenken for additional observations at Effelsberg, Jens Kauffmann for help with the $30 \mathrm{~m}$ observations, Marian Szymczak for trying to detect $\mathrm{CH}_{3} \mathrm{OH}$ emission towards UYSO 1 at Toruń, Philippe Salomé and Robert Zylka, both at IRAM Grenoble, for support in analyzing the PdBI data, as well as Henrik Beuther for helpful discussions. We would like to thank the referee, John Bally, for his helpful comments. T. S. is grateful for support through the A. V. Humboldt Foundation during his stay at the University of Hawaii. R.K. acknowledges support through Spitzer grant JPL no. 1276999. Partly based on Director's Discretionary Time observations collected at the Centro Astronómico Hispano Alemán (CAHA) at Calar Alto, operated jointly by the Max-Planck Institut für Astronomie and the Instituto de Astrofísica de Andalucía (CSIC). The National Radio Astronomy Observatory (NRAO) is operated by Associated Universities, Inc., under a cooperative agreement with the National Science Foundation. Partly based on observations carried out with the IRAM Plateau de Bure Interferometer and the IRAM $30 \mathrm{~m}$ telescope. IRAM is supported by INSU/CNRS (France), MPG (Germany) and IGN (Spain). Partly based on observations made with ESO Telescopes at the Paranal Observatory under programme IDs 074.C-0648(A) and 076.C-0773(B). The James Clerk Maxwell Telescope is operated by The Joint Astronomy Centre on behalf of the Science and Technology Facilities Council of the United Kingdom, the Netherlands Organisation for Scientific Research, and the National Research Council of Canada. This work is based in part on observations made with the Spitzer Space Telescope, which is operated by the Jet Propulsion Laboratory, California Institute of Technology under a contract with NASA. Partly based on observations with the 100-m telescope of the MPIfR (Max-Planck-Institut für Radioastronomie) at Effelsberg.

\section{References}

Benson, P. J., Caselli, P., \& Myers, P. C. 1998, ApJ, 506, 743

Beuther, H., Schilke, P., Gueth, F., et al. 2002a, A\&A, 387, 931

Beuther, H., Schilke, P., Sridharan, T. K., et al. 2002b, A\&A, 383, 892

Beuther, H., Schilke, P., \& Stanke, T. 2003, A\&A, 408, 601

Beuther, H., Schilke, P., Menten, K. M., et al. 2005, ApJ, 633, 535

Beuther, H., Churchwell, E. B., McKee, C. F., \& Tan, J. C. 2007, in Protostars and Planets V, ed. B. Reipurth, D. Jewitt, \& K. Keil, 165

Beuther, H., Semenov, D., Henning, T., \& Linz, H. 2008, ApJ, 675, L33

Claria, J. J. 1974, AJ, 79, 1022

Davis, C. J., Varricatt, W. P., Todd, S. P., \& Ramsay Howat, S. K. 2004, A\&A, 425,981

Forbrich, J., Schreyer, K., Posselt, B., Klein, R., \& Henning, T. 2004, ApJ, 602, 843, Paper I

Güsten, R., Nyman, L. Å., Schilke, P., et al. 2006, A\&A, 454, L13

Henning, T., Cesaroni, R., Walmsley, M., \& Pfau, W. 1992, A\&AS, 93, 525

Henning, T., Schreyer, K., Launhardt, R., \& Burkert, A. 2000, A\&A, 353, 211

Hollenbach, D. J., \& Shull, J. M. 1977, ApJ, 216, 419

Hollenbach, D. J., \& Tielens, A. G. G. M. 1999, Rev. Mod. Phys., 71, 173

Houk, N., \& Smith-Moore, M. 1988, in Michigan Spectral Survey, Ann Arbor, Dept. of Astronomy, Univ. Michigan, 4

Klein, R., Posselt, B., Schreyer, K., Forbrich, J., \& Henning, T. 2005, ApJS, 161, 361

Krumholz, M. R., \& McKee, C. F. 2008, Nature, 451, 1082

Linz, H., Stecklum, B., Henning, T., Hofner, P., \& Brandl, B. 2005, A\&A, 429, 903

Motte, F., \& André, P. 2001, A\&A, 365, 440

Palla, F., \& Stahler, S. W. 1992, ApJ, 392, 667

Rieke, G. H., Young, E. T., Engelbracht, C. W., et al. 2004, ApJS, 154, 25

Ryter, C. E. 1996, Ap\&SS, 236, 285

Schmidt-Kaler, T. 1982, in Landolt-Börnstein New Series, Group 6, 2b, ed. K. Schaifers, \& H.-H. Voigt, 227, 1

Scoville, N. Z., Sargent, A. I., Sanders, D. B., et al. 1986, ApJ, 303, 416

Shepherd, D. S., \& Churchwell, E. 1996, ApJ, 472, 225

Stanke, T., McCaughrean, M. J., \& Zinnecker, H. 2002, A\&A, 392, 239

Tafalla, M., Myers, P. C., Caselli, P., \& Walmsley, C. M. 2004, A\&A, 416, 191

Tafalla, M., \& Santiago, J. 2004, A\&A, 414, L53

Vuong, M. H., Montmerle, T., Grosso, N., et al. 2003, A\&A, 408, 581

Weigelt, G., Beuther, H., Hofmann, K.-H., et al. 2006, A\&A, 447, 655

Womack, M., Ziurys, L. M., \& Wyckoff, S. 1991, ApJ, 370, L99

Wu, Y., Zhang, Q., Chen, H., et al. 2005, AJ, 129, 330

Yorke, H. W., \& Bodenheimer, P. 2008, in Massive Star Formation: Observations Confront Theory, ed. H. Beuther, H. Linz, \& T. Henning, ASP Conf. Ser., 387, 189 